\title{
Test Time Reduction for Scan-Designed Circuits by Sliding Compatibility ${ }^{\dagger}$
}

\author{
Jau-Shien Chang Chen-Shang Lin \\ Department of Electrical Engineering \\ National Taiwan University \\ Taipei, Taiwan, R.O.C.
}

\begin{abstract}
$A$ post generation method for test time reduction of scan-designed circuits is developed in this paper. Maximum overlapping condition between consecutive applied patterns is identified. The application of the condition facilitated with the developed active sliding compatibility process significantly reduces the number of test clocks. It is demonstrated that the test clocks can be reduced by $50 \%$ on average from given test sets. Further evaluation shows that, for parity-scan, the test clocks required by our developed method are only $41 \%$ of those in [3].
\end{abstract}

\section{Introduction}

To increase the fault coverage and the test quality of sequential circuits, the now popular scan design had been proposed [12]. The main feature of scan design is that, by scanning the flip-flops, the complicated sequential test problem is transformed into the much simpler combinational one, thereby the desirable test quality can be achieved. However, the necessity to shift test patterns and responses in the long scan chain may incur significant increase in test application time as well as test cost. To minimize the additional testing time while retaining test quality of scan design, various scan clock reduction methods have been proposed $[1-5,7]$.

These previous works of test time reduction for scandesigned circuits can be divided in to the following two general approaches: reduction during test generation or after test generation. To reduce the test application time during test generation, one way is to generate a combinational test set as compact as possible. Several effective techniques have been developed to generate such compacted test sets[6,8-9]. However, as pointed out in [3], test compaction alone is not enough to reduce test application time because further reductions can always be obtained by carefully rearranging the test patterns. Alternatively, in $[4,7]$, the test generation is made to switch between scan-mode and nonscan-mode, and the scan mode is used only to achieve the required fault coverage. However, for circuits with many sequential hard-to-detected faults, the scan operations will occur frequently and result in test time worse than that of full-scan[4]. Moreover, complicated sequential test generation is involved in the process with the consequence of prolonged generation time.

The post generation approach is characterized by reducing scan requirements from a given test set. In $[1,2]$, based on the assumption that scan-in and scan-out flipflops are disjoint, each of the scan-in patterns is divided into several segments first. Then, the application of a test pattern to the CUT is invoked after scanning in only a segment but not necessarily a complete pattern. Thus, some faults originally detected by the later patterns may

TThis work was supported in part by the National Science Council under contract number NSC-83-0404-E-002-055 become detectable by these segmented patterns and the test time can be reduced due to pattern removal. More elaborate pattern-overlapping techniques have also been developed in [7]. The overlapping of successive pattern8 are obtained by precisely controlling the scan operation through the utilisation of don't-care bits in the scanned flip-flops. However, for a given test set with few don'tcare bits, the overlapping often collapses due to the minor difference between successive patterns. In summary, the effectiveness of these previous works is heavily restricted by the characteristics of the given test set.

Recently, a novel idea, parity-scan design, has been proposed in [3], in which an extra parity output is introduced to the scanned flip-flops to enhance the observability. With this simple parity output, the scan operation can be eliminated if the fault effects can be easily observed and the content of flip-flops can be reused. To exploit the enhanced facility, direct test time reduction has been considered in [3] by generating a pattern with part of the previous pattern as the constraint so that a test set with highly overlapping patterns can be obtained. As shown in [3], the test time is reduced by $30 \%$ on average with the modified FAN[11]. However, the high overlapping among patterns is obtained at the expense of a much larger test set and as a result, even with the parity output, the reduction in test time is often inferior to that by compacting the test set for pure scan design. From the observations on these previous works, it can be seen that, in order to obtain consistently good results in test application time reduction, post generation approach with a compact test set is preferable.

We are going to show in this paper that more significant reduction of test clocks in scan-designed circuits can be achieved by the post generation approach. Namely, the reduction is performed after an initial test set is generated. We identify the condition which allows the maximum overlapping between successive applied patterns and then actively modify these patterns to exploit the overlopping without sacrificing fault coverage. The experimental results on 22 ISCAS89 benchmark circuits show that, for the test sets generated by PODEM, $50 \%$ test clocks in pure full-scan can be reduced on average. Furthermore, for the parity-scan design proposed in [3], our method needs only $52 \%$ test clock cycles of those generated in [3]. When the given test set is already compact, the improvement over [3] is even more significant, only $41 \%$ test clocks are required. The reduction is achieved strictly on the domain of combinational circuit.

The paper is organired as follow. Some notations and definitions about scan design are introduced in Section 2. The proposed Maximum Overlapping condition is described in Section 3. In Section 4, the active operation of sliding compatibility for maximum overlapping is introduced. Section 5 provides the Experimental Results. The Conclusions are given in the last Section. 


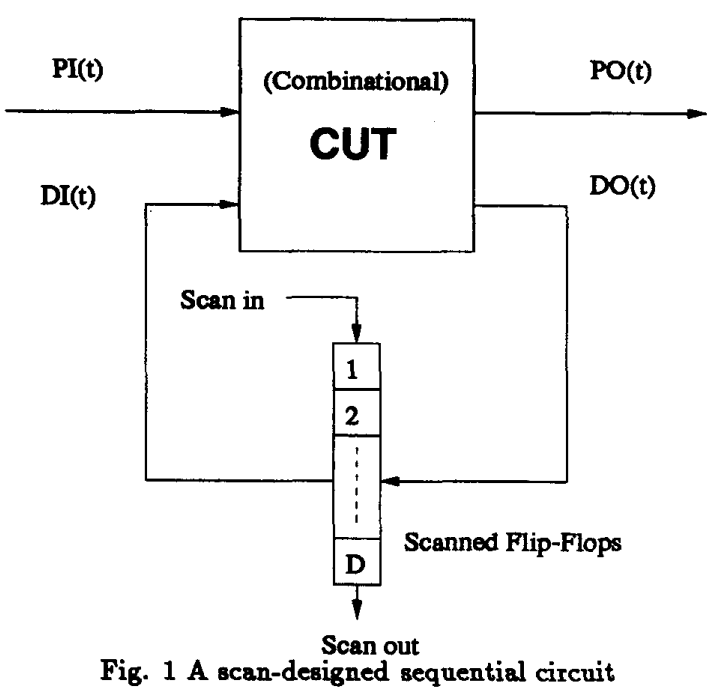

\section{Scan Design}

In this section, the full scan-designed circuits will be briefly discussed. Some notations and definitions useful for subsequent discussions will also be introduced.

For a scan-designed circuit as shown in Fig. 1, each test pattern $t$ for the CUT (Circuit Under Test) consists of two parts: the part applied to PI denoted as $P I(t)$ and the other part for the FFs (Flip-Flops) as $D I(t)$. In the test application of $t, D I(t)$ must first be shifted in to the scan path which generally consists of all FFs in the circuit. Let $R E S P(t)$ be the response of CUT after applying $t$. $R E S P(t)$ can be similarly divided into $P O(t)$ and $D O(t)$, where $P O(t)$ is the response appearing at $P O$ and $D O(t)$ is that to be loaded into FFs. $D O(t)$ must also be shifted out of the scan path for observation, which may overlap with the scan-in of the next pattern.

Let $T$ be the test set to be applied and $D$ be the number of shifts for each pattern (in general, $D$ is the number of scanned flip-flops), then total testing time, $t_{T}$, is

$$
t_{T}=(|T|+1) * D * S+|T| * C
$$

where $S$ and $C$ are the periods of a shifting clock and system clock, respectively. Assuming $S=C, t_{T}$ becomes $t_{T}=((|T|+1) * D+|T|) * S$

The total test clocks of $T$ for a scan-designed circuit, $T T C$, is then

$$
T T C=(|T|+1) * D+|T|
$$

From (2.3), it can be seen that, to reduce the total test clocks, we can either compact the test set $T$ as small as possible or shorten the number of shifts for each pattern. For test set compaction, there already exist effective tools such as COMPACTEST'[9] and TSR[6]. At present, TSR has been adopted in our work. Therefore, in this paper, we will aim at how to reduce the number of test clocks on $D$. Note that we assume the scan-chain topology has been determined before test clock reduction.

\section{Maximum Overlapping}

Given a test set, the primary step of test clock reduction is to identify the maximum overlapping possible between two consecutive patterns without sacrificing fault coverage. In this section, the condition for Maximum Overlapping will be discussed.
Since the purpose of reduction is to use the current content of FFs as part of the next scan-in pattern, two types of overlapping are possible, either overlapping with the response of the previous pattern or the previous pattern itself. Both need some elaboration. In the first case, one must be sure that the content of FFs is not contaminated by the fault effect of all possible faults under consideration. And in the second case, the fault effect should be observed directly from primary outputs and the FFs retain the previous pattern by skipping the load response operation.

Two special cases for pattern overlapping have been proposed in [3]. Under the constraint that all the activated faults of the current applied pattern $t_{i}$ can be observed at $\mathrm{PO}$, the following two cases are accepted for reducing the test clocks of the next pattern $t_{i+1}$.

case (a) Complete DO-reuse. $D O\left(t_{i}\right) \equiv D I\left(t_{i+1}\right)$,

case (b) Complete DI-reuse: $D I\left(t_{i}\right) \equiv D I\left(t_{i+1}\right)$.

The ' $\equiv$ ' above is the compatible relation. Two vectors are said to be compatible if all the corresponding bits are either of the same logic value or one of them is ' $x$ ' (don't care). For example, the two vectors, $v 1=(0 x 01)$ and $v 2=(010 x)$, are compatible and denoted as $v 1 \equiv v 2$. When one of the above two cases is satisfied, the current content of FFs can be completely reused as part of $t_{i+1}$ and the scan-in operation of $t_{i+1}$ can be eliminated. However, the conditions used in [3] are clearly restrictive. The general conditions for maximum overlapping will be described next.

To allow maximum overlapping, we will first examine the conditions by which the content of FFs can be safely reused. As discussed in the beginning of this section, to reuse the DO-part, one must be sure that no fault effect will appear in FFs. In other words, the content of FFs must be the fault-free response to allow safely resue. This is by no means trivial since the fault effect of some faults can only be observed after scanning out the content of FFs. Nevertheless, we generally need not scan out the whole chain to determine whether or not it is the faultfree response. This is stated in the following observation.

Observation 1 (DO-Reuse): Under single fault assumption, after applying a pattern t to the CUT and loading its response $D O(t)$ into the scanned FFs, the presence of a detectable fault $f$ of $t$ can be determined from either $P O s$ or its first fault effect bit in $D O(t)$. Furthermore, if $P O$ s and the first fault effect bits of all detectable faults of $t$ in $D O(t)$ have been observed to be fault-free, then these faults are not present and $D O(t)$ is the fault-free response.

The implication of Observation 1 is that after examining a few possible fault-effect bits in $D O(t)$, the remaining fault-free response may provide an overlapping chance with the next pattern. From the observation, the number of bits to be shifted out after applying a pattern is then $M A X(M I N B I T(f))$ for each detectable fault $f$ by the pattern. This number can be easily determined by the fault simulation before actual test application. After shifting out this predetermined number of bits, it can be decided whether or not the remaining bits are fault-free and can be safely reused. For the example shown in Table 1 , suppose three faults $\{f 1, f 2, f 3\}$ are detected by applying pattern $t$ and the fault-free $D O(t)=(1001010)$, by means of possible fault-effect bits computation in $D O(t)$, the faulty bit nearest to the scan-out pin is selected as $M I N B I T(f)$. Since the maximum of MIN BIT is found to be $4, D O(t)$ must be shifted out 4 bits before re-usage. 


\begin{tabular}{|c|c|c|}
\hline & $D O(t)$ & \\
& 7654321 & $M I N B I T(f)$ \\
\hline$f f$ & 1001010 & - \\
\hline$f 1$ & $d 001 d 10$ & 3 \\
\hline$f 2$ & $1 d 0 d 010$ & 4 \\
\hline$f 3$ & $1 d 0 d 0 d 0$ & 2 \\
\hline
\end{tabular}

$f f$ : Fault Free response

$d$ : denote the fault effect

Table 1. Example of $M A X(M I N B I T(f))$ Computation

The DI-reuse condition is the same as described earlier and formally given as follows.

Observation 2 (DI-Reuse): The current pattern in the $F F s, D I\left(t_{i}\right)$, may be part of $D I\left(t_{i+1}\right)$ only if all the detected faults of $t_{i}$ can be observed at POs alone.

It can be seen that when fault effects must be observed from FFs, the DI-reuse can not be applied. The problem can be aignificantly alleviated if extra hardware is used to enhance observability such as that in parity-scan designed circuits[3].

The above two observations are the necessary conditions to reuse the current content of FFs. The following observation provides a sufficient condition for overlapping the next pattern with the current content of FFs, so that the test clocks can be reduced.

Observation 3 (Maximum Overlapping): The current content of $F F s, S F F\left(t_{i}\right)\left(D I\left(t_{i}\right)\right.$ or $\left.D O\left(t_{i}\right)\right)$, may constitute part of $D I\left(t_{i+1}\right)$ without sacrificing fault coverage if the conditions of Observations 1 or 2 are satisfied and $T A I L\left(S F F\left(t_{i}\right)\right) \equiv H E A D\left(D I\left(t_{i+1}\right)\right)$, where $T A I L$ and $H E A D$ are the trailing and leading parts during scan, respectively.

The last condition of Observation 3 requires only the trailing part of SFF $\left(t_{i}\right)$ is compatible with the leading part of $D I\left(t_{i+1}\right)$ without explicitly specifying the size of parts. The condition is named sliding compatibility of $S F F\left(t_{i}\right)$ and $D I\left(t_{i+1}\right)$. Sliding compatibility is the generalisation of complete compatibility for test clock reduction.

The extent of overlapping which can be actually achieved from the above observation depends on the ordering of scan chain. Although it is theoretically possible to rearrange scan path to obtain maximum reduction, physical constraints discourage such arbitrary rearrangement. Hence the aspect of rearrangement is not discussed and, in our experiment, the natural ordering of scan path is used.

Based on Observation 3, the process for maximum overlapping can be performed as follow. In the previous example in Table 1, after shifting 4 bits out of $D O(t)=$ $(1001010)$, there are still 3 bits can be overlapped with the next pattern. In other words, three scan clocks can be reduced if the next pattern is compatible with (xxxx100). To take fullest advantage of this situation, the next pattern can be chosen accordingly. When this is not possible, the next choice can be the one that is sliding compatible with (xxx 100), i.e., compatible with (xxxx10) and then with ( $x \times x \times x 1$ ). Note that, with sliding compatibility, the extent of test clock reduction decreases step by step. In the extreme case, not even sliding compatibility is possible and regular scan operation must be performed. A more effective implementation of this process will be described in the next section.

\section{Active Sliding Compatibility}

The goal of maximum overlapping is to optimally reuse the current content of scanned FFs in the next scan-in operation. However, for a test set lack of sliding compatibility between patterns, the reduction on test clock will not be significant. It means that the effectiveness of maximum overlapping could be restricted by the nature of the given test set. Therefore, to further enhance the reuse in maximum overlapping, we propose the process, Active $S$ liding Compatibility, which actively modifies the test set for sliding compatibility and increase the chance of reuse while keeping the overall fault coverage intact.

The basic idea of active sliding compatibility is to take advantage of the over-specification property of the given test set. For example, for a pattern $t=(01111)$ in a test set $T$, the fault coverage of $T$ may still keep intact when $t$ is changed to $t^{\prime}=(0 x 1 x 1)$. In this case, $t$ is said to be overspecified in $T$ and these bits changed from ' 0 ' or ' 1 ' to ' $x$ ' are said to be raised. Comparing $t$ with $t^{\prime}, t^{\prime}$ obvioualy has more chances to be compatible with any arbitrary vector than $t$. Thus, when trying to reuse the current contents of FFs for the scan-in of $t$, modifying $t$ to $t^{\prime}$ would be a better choice.

In active sliding compatibility, for the next pattern to be scanned in, those sliding incompatible bits with the current contents of FFs will be modified (raised) to ' $\boldsymbol{x}$ ' for re-usage. However, during raising these incompatible bits, it is essential to preserve the overall fault coverage, $D E T(T, F)$. The following observation allows us to perform the raising operation while keeping the fault coverage intact[6]. Define $E S S_{T}(t, F)$, the essential faults of $t$, as the set of faults in $F$ that can only be detected by $t$ but not others in $T$.

Observation 4: Given a fault set $F$ and a test set $T$ of $F$, for a pattern $t \in T$, if $t$ is substituted by $t$ ' such that $D E T\left(\left\{t^{\prime}\right\}, F\right) \supseteq E S S_{T}(t, F)$, then $T^{\prime}=T-\{t\}+\left\{t^{\prime}\right\}$ has at least the same amount of fault coverage as $T$.

The implication of Observation 4 is that active sliding compatibility can be performed by raising some bits of $t$ while monitoring the detectability of $E S S_{T}(t, F)$. And, the detectability of $E S S_{T}(t, F)$ can easily verified by fault simulation.

\begin{tabular}{|l|c|c|c|c|}
\hline Step & DI(t) & $\begin{array}{c}\text { Contents } \\
654321\end{array}$ & Operation & $\equiv D I\left(t_{1}\right) ?$ \\
\hline Init & $t_{1}$ & 101000 & - & - \\
& $t_{2}$ & 010011 & - & No \\
& $t_{3}$ & 111110 & - & No \\
\hline 1 & $t_{1}$ & 101000 & - & - \\
& $t_{2}$ & $x 1 \times 010$ & bit raising & No \\
& $t_{3}$ & $1 \times 1110$ & bit raising & No \\
\hline 2 & $t_{1}$ & $x 10100$ & shift out 1 bit & - \\
& $t_{2}$ & $010 x x 1$ & bit raising & No \\
& $t_{3}$ & $11 \times 110$ & bit raising & No \\
\hline 3 & $t_{1}$ & $x x 1010$ & shift out 2 bits & - \\
& $t_{2}$ & $01 \approx 011$ & bit raising & No \\
& $t_{3}$ & $111 x 10$ & bit raising & Yes \\
\hline
\end{tabular}

Table 2. Example of active aliding compatibility

With Observation 4, the brief procedure for maximum overlapping described at the last of Section 3 can be refined to the active sliding compatibility process for achieving more reductions on test clocks. The process is illustrated by the following example. As shown in Table 2, for 
a test set $T=\left\{t_{1}, t_{2}, t_{3}\right\}$, after applying $t_{1}$, active sliding compatibility is performed to select the next applied pattern. Only DI-reuse is considered in this example for simplicity. Initially, $D I\left(t_{1}\right), D I\left(t_{2}\right)$ and $D I\left(t_{3}\right)$ are mutually incompatible. In step 1 , after the raising operation on these incompatible bits, the DI-parts of the three patterns are still incompatible. Therefore, one shift ont operation is performed on $D I\left(t_{1}\right)$. In step 2, after the $D I\left(t_{1}\right)$ is shifted out 1 bit, active operations for compatibility is performed on $D I\left(t_{2}\right)$ and $D I\left(t_{3}\right)$ again. Unfortunately, it also fail8. In step 3, after the content of $D I\left(t_{1}\right)$ is shifted out one more bit, the DI-parts of $t_{1}$ and $t_{3}$ become compatible after raising. Thus, 4 scan clocks can be saved for the scan-in of $t_{3}$. Without these active raising operations, only one scan clock (choosing $t_{2}$ as the next pattern) can be reduced in this example.

\section{Experimental Results}

To show the effectiveness of our method (ACT), these test clock reduction techniques proposed in this paper have been implemented on SUN4-SPARC2 workstation and 22 ISCAS'89 benchmark circuits are evaluated.

\begin{tabular}{|c|c|c|c|c|c|}
\hline Ckts & SFF & PDM & TSR & PDM-P & TSR-P \\
\hline 8208 & 8 & 50 & 27 & 50 & 27 \\
\hline 8298 & 14 & 51 & 25 & $\mathbf{5 1}$ & 24 \\
\hline 8344 & 15 & 33 & 14 & 33 & 15 \\
\hline 8349 & 15 & 33 & 14 & 33 & 15 \\
\hline 8382 & 21 & 50 & 25 & 49 & 25 \\
\hline 8386 & 6 & 97 & 63 & 92 & 63 \\
\hline 8420 & 16 & 88 & 44 & 88 & 44 \\
\hline 8444 & 21 & 47 & 25 & 47 & 25 \\
\hline 8510 & 6 & 79 & 57 & 76 & 56 \\
\hline 8526 & 21 & 98 & 50 & 97 & 50 \\
\hline 8641 & 19 & 88 & 24 & 98 & 24 \\
\hline 8713 & 19 & 88 & 24 & 85 & 24 \\
\hline $\mathbf{8 8 2 0}$ & 5 & 188 & 96 & 183 & 96 \\
\hline 8832 & 5 & 180 & 97 & 182 & 96 \\
\hline 8838 & 32 & 155 & 76 & 152 & 76 \\
\hline 8953 & 6 & 109 & 76 & 115 & 77 \\
\hline 81196 & 17 & 222 & 124 & 232 & 126 \\
\hline 81238 & 17 & 230 & 133 & 234 & 129 \\
\hline 81423 & 74 & 126 & 32 & 118 & 32 \\
\hline 81488 & 6 & 176 & 104 & 183 & 106 \\
\hline 81494 & 6 & 185 & 102 & 175 & 103 \\
\hline 85378 & 179 & 425 & 109 & 415 & 111 \\
\hline
\end{tabular}

SFF: number of scanned flip-flope

- number of total flip-flops

Table 3. Test sets of ISCAS89 benchmark circuits

In Table 3, the number of Scanned Flip-Flops (SFF) of these circuits are shown. In addition, two initial test sets of these circuits are evaluated for both full-scan and parityscan version. One initial test set is generated by a simple PODEM-like ATPG and the other is its highly compacted set by TSR[6]. These two test sets can be regarded as two extremes for an arbitrarily given test set. The sizes of these four different test sets, two for full scan and two for parity-scan [3] are also included in Table 3. For the test sets in PDM-P and TSR-P columns, they are generated in a similar way to PDM and TSR test sets except a paritychecking path for pseudo POs (i.e. scanned FFs) and an extra PARITY output are added during test generation. In Table 4 and 5 , the test clock reduction results of ACT on the PDM, TSR, PDM-P, TSR-P test sets are shown.
The comparison of ACT with previous works are provided in Table 6 .

In Table 4, the test clocks generated by ACT for PODEM and TSR test sets are given. The final results are shown in the ACT column and those only with sliding but without bit-raising operations are listed in the SLIDE column. The number after each datum is the normalized ratio with respect to pure full scan (FSCAN) of the given test set. For the PODEM test sets, by the sliding operation (SLIDE), $29 \%$ test clock reduction can be achieved on average as shown in the last row. It can be seen that SLIDE is more effective for those circuits with less scanned flip-flops such as 8820, s832, s1488 and s1494. For those circuits with a large amount of scanned FFs such as 81494 and 85378, the reduction is less successful due to the difficulty of overlapping between successive scanned patterns. However, when bit-raising is added to increase the chance of overlapping, as shown in the ACT column, up to $46 \%$ and $65 \%$ reduction on test clocks for 81423 and 85378 can be obtained respectively. This shows the effectiveness of the proposed active sliding compatibility process. On average, by ACT, $50 \%$ of test clocks for FSCAN can be reduced. For the TSR test sets, although the test sets are much more compact than those of PODEM and hence there is less chance for overlapping, $27 \%$ reduction can still be achieved by our methods. When comparing the total test clocks of FSCAN for TSR test sets with those for PODEM test set$s$ in the $\sum / A V G$ row, less than $1 / 3(38268 / 118036)$ of the test clocks are required, a very significant reduction on test clocks and test cost. It demonstrates that nsing a compact test set as the starting point of test clock reduction is worthwhile and the efforts on the additional test set compaction can be well-justified.

For the parity-scan designed circuits, the results of $A C$ T on PODEM-P and TSR-P test sets are shown in Table 5 . In comparison with Table 4, with the extra parity output, the average reduction ratio can be increased from $50 \%$ to $72 \%$ for PODEM test sets and from $27 \%$ to $53 \%$ for TSR test sets. This shows the positive effect of enhanced observability by the parity output. Note that, for these circuits with more scanned FFs such as 8838, s1423 and s5378, the results of ACT on TSR and TSR-P test sets are quite close $((1163,1013),(2285,2086),(14252,14351))$. The reason is as follows. Due to with a large number of FFs and compacted test sets, the fault effect of each fault in these circuits are more likely to reach many FFs and scan operations for each pattern generally can not be avoided. However, from Observation 1 , we are able to observe the existence of these faults through a few shift-out operations almost as effective as adding an extra parity output. It suggests that, in those circuits with large number of flipflops, it is more advantageous to use the proposed ACT starting from the compacted test set since it can accomplish almost the same amount of test clock reduction as the additional parity chain, albeit without any hardware overhead.

The comparison of ACT with the previous work, ParityScan [3], is shown in Table 6. The test clocks by [3] are listed in the PSCAN column. The results of ACT with the parity output are shown in the ACT-PDM(P) and ACTTSR(P) column respectively, which are identical to Table 5. The number after each datum is the normalised ratio with respect to PSCAN. Comparing ACT-PDM(P) and PSCAN, except s1196 and 81238, our results are far superior to those of [3]. On average, the test clocks of ACTPDM(P) are only 52\% of those of PSCAN. It clearly shows effectiveness of the proposed active test clock reduction. In particular, for 81423 and $85378,61 \%$ and $75 \%$ reduction can be achieved, even though the test set size used in [3] are approximately equal to that of the PODEM-P test set shown in Table 3 . The result demonstrates the merit of 


\begin{tabular}{|c|c|c|c|c|c|c|}
\hline & \multicolumn{3}{|c|}{ PODEM test sets } & \multicolumn{3}{|c|}{ TSR test sets } \\
\hline Clkts & FSCAN & SLIDE & ACT & FSCAN & SLIDE & ACT \\
\hline 8208 & 458 & $301 / 0.66$ & $220 / 0.48$ & 251 & $198 / 0.79$ & $182 / 0.73$ \\
\hline $\mathbf{8 2 9 8}$ & 779 & $654 / 0.84$ & $478 / 0.61$ & 389 & $354 / 0.91$ & $317 / 0.81$ \\
\hline 8344 & 543 & $471 / 0.87$ & $360 / 0.66$ & 239 & $225 / 0.94$ & $224 / 0.94$ \\
\hline 8349 & 543 & $471 / 0.87$ & $360 / 0.66$ & 239 & $229 / 0.96$ & $227 / 0.95$ \\
\hline 8382 & 1121 & $1001 / 0.89$ & $728 / 0.65$ & 571 & $535 / 0.94$ & $504 / 0.88$ \\
\hline 8386 & 685 & $418 / 0.61$ & $405 / 0.59$ & 447 & $341 / 0.76$ & $341 / 0.76$ \\
\hline 8420 & 1512 & $1110 / 0.73$ & $582 / 0.38$ & 764 & $638 / 0.84$ & $431 / 0.56$ \\
\hline 8444 & 1055 & $937 / 0.89$ & $638 / 0.60$ & 571 & $545 / 0.95$ & $448 / 0.78$ \\
\hline 8510 & 559 & $333 / 0.60$ & $320 / 0.57$ & 405 & $273 / 0.67$ & $276 / 0.68$ \\
\hline 8526 & 2177 & $1905 / 0.88$ & $1293 / 0.59$ & 1121 & $1039 / 0.93$ & $947 / 0.84$ \\
\hline 8641 & 1779 & $1277 / 0.72$ & $645 / 0.36$ & 499 & $458 / 0.92$ & $370 / 0.74$ \\
\hline 8713 & 1779 & $1293 / 0.73$ & $624 / 0.35$ & 499 & $475 / 0.95$ & $367 / 0.74$ \\
\hline 8820 & 1133 & $680 / 0.60$ & $633 / 0.56$ & 581 & $474 / 0.82$ & $477 / 0.82$ \\
\hline 8832 & 1085 & $657 / 0.61$ & $623 / 0.57$ & 587 & $479 / 0.82$ & $493 / 0.84$ \\
\hline 8838 & 5147 & $3667 / 0.71$ & $1872 / 0.36$ & 2540 & $2101 / 0.83$ & $1163 / 0.46$ \\
\hline 8953 & 769 & $446 / 0.58$ & $428 / 0.56$ & 538 & $373 / 0.69$ & $377 / 0.70$ \\
\hline 81196 & 4013 & $1610 / 0.40$ & $726 / 0.18$ & 2249 & $1045 / 0.46$ & $576 / 0.26$ \\
\hline .1238 & 4157 & $1552 / 0.37$ & $745 / 0.18$ & 2411 & $1161 / 0.48$ & $676 / 0.28$ \\
\hline 51423 & 9524 & $8850 / 0.93$ & $5109 / 0.54$ & 2474 & $2429 / 0.98$ & $2285 / 0.92$ \\
\hline 81488 & 1238 & $747 / 0.60$ & $711 / 0.57$ & 734 & $587 / 0.80$ & $587 / 0.80$ \\
\hline 81494 & 1301 & $770 / 0.59$ & $743 / 0.57$ & 720 & $591 / 0.82$ & $591 / 0.82$ \\
\hline .5378 & 76679 & $69792 / 0.91$ & $26535 / 0.35$ & 19799 & $19618 / 0.99$ & $14252 / 0.73$ \\
\hline$\sum / A V G$ & $118036 / 1.00$ & $98942 / 0.71$ & $44778 / 0.50$ & $38628 / 1.00$ & $34168 / 0.83$ & $26111 / 0.73$ \\
\hline
\end{tabular}

FSCAN: test clocks of pure Full Scan

SLIDE: ACT without bit-raising

ACT: Active Sliding Compatibility technique

Table 4. Results of test clock reduction by ACT

post generation approach of test clock reduction. It is also interesting to compare PSCAN with ACT-TSR(P). In each evaluated case, the result of ACT-TSR(P) is far better than that of PSCAN. The average ratio of test clocks is only $41 \%$. Recall that, in [3], the test sets are generated by preserving as more common parts as possible among patterns to increase the chance of overlapping. Evidently, the compactness of the generated test set is scarified. This comparison result clearly shows that scarifying the compactness of the test set for overlapping is not worthwhile and a compact test set is desired for test clock reduction in scanned design.

In the last column of Table 6, the CPU-time for ACTPDM(P) is shown. Except 85378 , all the examples can be completed in one minute. For 85378, due to its circuit size, large test set and scanned FFs, more CPU-time, albeit not prohibitively long, is required. For ACT-TSR(P), because the test sets are much more compact than those of ACTPDM(P), the CPU-time is far less than that shown in the Table. For example, in ACT-TSR(P), the CPU-time for 85378 is only 448 seconds.

\section{Conclusions}

Test time reduction for scan-designed circuits has been investigated in this paper. To reduce the lengthy shifting operations in the long scan path, post generation method to optimally reuse the contents of the scanned flip-flops have been developed. From the experimental results on 22 ISCAS89 benchmark circuits, up to $50 \%$ test clocks can be reduced on average by the proposed method. Furthermore, when the parity output is included in the scan-designed CUT, the resultant test clocks by ACT have been only $41 \%$ of those in [3]. It has been reported that by switching be- tween scan-mode and nonscan-mode, further reduction on test clock cycles are possible in pure scan designed circuits. However, a sequential circuit test generation will then be required and the test generation time will be substantially increased. To retain the advantage of simple combinational test generation of scan-designed circuits, the modeswitching is not performed in this work.

\section{References}

[1] M. S. Abadir and M. A. Breuer, "Scan Path With Look Ahead Shifting (SPLASH)," Proceedings of 1986 International Test Conference, pp. 696-704.

[2] M. S. Abadir, "Efficient Scan Path Testing Using Sliding Parity Response Compaction, ${ }^{\text {Proceed }}$ ings of 1987 International Test Conference, pp. 332-335.

[3] H. Fujiwara and A. Yamamoto, "Parity-Scan Design to Reduce the Cost of Test Application," IEEE Trans. Computer Aided Design, Vol. 12, No. 10, pp. 1604-1611, Oct. 1993.

[4] S. Y. Lee and K. K. Saluja, "An Algorithm to Reduce Test Application Time in Full Scan Designs," Proceedings of 1992 ICCAD, pp. 17-20.

[5] I. Pomeranz and S. M. Reddy, "A Test Application Scheme for Embedded Full-Scan Circuits to Reduce Testing Costs," Proceedings of First Asion Test Symposium, Pp. 206-211, 1992. 


\begin{tabular}{|c|c|c|c|c|c|c|}
\hline & \multicolumn{3}{|c|}{ PODEM-P test sets } & \multicolumn{3}{|c|}{ TSR-P test sets } \\
\hline Ckts & FSCAN & SLIIE & ACT & FSCAN & SLIDE & $\mathbf{A C T}$ \\
\hline$\$ 208$ & 458 & $296 / 0.65$ & $222 / 0.48$ & 251 & $198 / 0.79$ & $183 / 0.73$ \\
\hline $\mathbf{8 9 8}$ & 779 & $534 / 0.69$ & $241 / 0.31$ & 374 & $282 / 0.75$ & $171 / 0.46$ \\
\hline 8344 & 543 & $409 / 0.75$ & $247 / 0.45$ & 255 & $213 / 0.84$ & $191 / 0.75$ \\
\hline 8349 & 543 & $409 / 0.75$ & $247 / 0.45$ & 255 & $213 / 0.84$ & $191 / 0.75$ \\
\hline $\mathbf{8 3 8 2}$ & 1099 & $865 / 0.79$ & $465 / 0.42$ & 571 & $485 / 0.85$ & $372 / 0.65$ \\
\hline 8386 & 650 & $198 / 0.30$ & $180 / 0.28$ & 447 & $152 / 0.34$ & $153 / 0.34$ \\
\hline 8420 & 1512 & $982 / 0.65$ & $415 / 0.27$ & 764 & $601 / 0.79$ & $366 / 0.48$ \\
\hline 8444 & 1055 & $872 / 0.83$ & $381 / 0.36$ & 571 & $495 / 0.87$ & $344 / 0.60$ \\
\hline .510 & 538 & $161 / 0.30$ & $161 / 0.30$ & 398 & $156 / 0.39$ & $155 / 0.39$ \\
\hline 8526 & 2155 & $1757 / 0.82$ & $5 5 6 \longdiv { 0 . 2 6 }$ & 1121 & $967 / 0.86$ & $538 / 0.48$ \\
\hline 8641 & 1979 & $1168 / 0.59$ & $329 / 0.17$ & 499 & $406 / 0.81$ & $265 / 0.53$ \\
\hline 8713 & 1719 & $1042 / 0.61$ & $306 / 0.18$ & 499 & $428 / 0.86$ & $289 / 0.58$ \\
\hline 8820 & 1103 & $249 / 0.23$ & $252 / 0.23$ & 581 & $169 / 0.29$ & $167 / 0.29$ \\
\hline 8832 & 1097 & $264 / 0.24$ & $263 / 0.24$ & 581 & $174 / 0.30$ & $183 / 0.31$ \\
\hline 8838 & 5048 & $2918 / 0.58$ & $1248 / 0.25$ & 2540 & $1896 / 0.75$ & $1013 / 0.40$ \\
\hline 8953 & 811 & $236 / 0.29$ & $211 / 0.26$ & 545 & $165 / 0.30$ & $165 / 0.30$ \\
\hline 81196 & 4193 & $1180 / 0.28$ & $404 / 0.10$ & 2285 & $575 / 0.25$ & $278 / 0.12$ \\
\hline $\mathbf{8 1 2 3 8}$ & 4229 & $1170 / 0.28$ & $408 / 0.10$ & 2339 & $617 / 0.26$ & $285 / 0.12$ \\
\hline$\$ 1423$ & 8924 & $7919 / 0.89$ & $3041 / 0.34$ & 2474 & $2324 / 0.94$ & $2086 / 0.84$ \\
\hline 81488 & 1287 & $313 / 0.24$ & $296 / 0.23$ & 748 & $221 / 0.30$ & $221 / 0.30$ \\
\hline 81494 & 1231 & $293 / 0.24$ & $279 / 0.23$ & 727 & $207 / 0.28$ & $207 / 0.28$ \\
\hline .5378 & 74879 & $60835 / 0.81$ & $19563 / 0.26$ & 20159 & $19363 / 0.96$ & $14351 / 0.71$ \\
\hline$\sum / A V G$ & $115832 / 1.00$ & $84070 / 0.54$ & $29715 / 0.28$ & $38984 / 1.00$ & $30307 / 0.62$ & $22174 / 0.47$ \\
\hline
\end{tabular}

Table 5. Results of test clock reduction by ACT with PARITY output

[6] J. S. Chang and C. S. Lin, "Test Set Compaction for Combinational Circuits," Proceedings of First Asian Test Symposium, pp. 20-25, 1992.

[7] W. C. Lai, C. P. Kung and C. S. Lin, "Test Time Reduction in Scan Design Circuits, ${ }^{\prime}$ Proceedings of $1993 E D A C$, pp. $489-498$.

[8] Gert-Jan Tromp, "Minimal Test Sets for Combinational Circuits," Proceedings of 1991 International Test Conference, pp. 204-209, 1991.

[9] I. Pomeranz, L. N. Reddy and Sudhakar M. Reddy, "COMPACTEST: A Method to Generate Compact Test Sets for Combinational Circuits," Proceedings of 1991 International Test Conference, pp. 194-203.

[10] P. Goel, “An Implicit Enumeration Algorithm to Generate Tests for Combinational Logic Circuits," IEEE Trans. on Computers, Vol. C-30, No. 3, Mar. 1981 .

[11] H. Fujiwara and T. Shimono, "On the acceleration of Test Generation Algorithms," IEEE Tran s. Computers, Vol. C-32, No. 12, pp. 1137-1144, Dec. 1983.

[12] E. B. Einchelberger and T. W. Williams, "A logic design structure for LSI testability," Proceeding of Design Automation Conference, 1977, pp. 462468.

\begin{tabular}{|c|c|c|c|c|}
\hline Ckts & PSCAN[3] & $\begin{array}{c}\text { ACT- } \\
\text { PDM(P) }\end{array}$ & $\begin{array}{c}\text { ACT- } \\
\text { TSR(P) }\end{array}$ & TIME \\
\hline 8208 & 279 & $222 / 0.80$ & $183 / 0.66$ & $\overline{2.0}$ \\
\hline 8298 & 495 & $241 / 0.49$ & $171 / 0.35$ & 3.0 \\
\hline 8344 & 461 & $247 / 0.54$ & $191 / 0.41$ & 3.0 \\
\hline 8349 & 443 & $247 / 0.56$ & $191 / 0.43$ & 3.0 \\
\hline 8382 & 828 & $465 / 0.56$ & $372 / 0.45$ & 4.0 \\
\hline 8386 & 380 & $180 / 0.47$ & $153 / 0.40$ & 5.0 \\
\hline 8420 & 1296 & $415 / 0.32$ & $366 / 0.28$ & 7.0 \\
\hline 8444 & 936 & $381 / 0.41$ & $344 / 0.37$ & 5.0 \\
\hline 8510 & 354 & $161 / 0.45$ & $155 / 0.44$ & 6.0 \\
\hline 8526 & 1707 & $556 / 0.33$ & $538 / 0.32$ & 10.0 \\
\hline 8641 & 579 & $329 / 0.57$ & $265 / 0.46$ & 12.8 \\
\hline 8713 & 729 & $306 / 0.42$ & $289 / 0.40$ & 12.1 \\
\hline 8820 & 487 & $252 / 0.52$ & $167 / 0.34$ & 20.1 \\
\hline 8832 & 471 & $263 / 0.56$ & $183 / 0.39$ & 20.7 \\
\hline 8838 & 5602 & $1248 / 0.22$ & $1013 / 0.18$ & 30.2 \\
\hline $8953^{*}$ & 2391 & $211 / 0.09$ & $165 / 0.07$ & 16.3 \\
\hline 81196 & 359 & $404 / 1.13$ & $278 / 0.77$ & 40.1 \\
\hline $\mathbf{8 1 2 3 8}$ & 351 & $408 / 1.16$ & $285 / 0.81$ & 44.7 \\
\hline 81423 & 7894 & $3041 / 0.39$ & $2086 / 0.26$ & 60.2 \\
\hline $\mathbf{s 1 4 8 8}$ & 617 & $296 / 0.48$ & $221 / 0.36$ & 36.8 \\
\hline 81494 & 702 & $279 / 0.40$ & $207 / 0.29$ & 35.0 \\
\hline 85378 & 76739 & $19563 / 0.25$ & $14351 / 0.19$ & 1492.0 \\
\hline$\sum$ & 101709 & 29504 & 22009 & - \\
\hline$A V G$ & 1.0 & 0.52 & 0.41 & - \\
\hline
\end{tabular}

ACT-PDM(P): results of ACT on PDM-P teat sets ACT-TSR(P): results of ACT on TSR-P test sets TIME: CPU time (sec) of ACT-PDM(P) on SUN4-SPARC2

*: removed from comparison due to different no. of SFF.

Table 6. Comparison of ACT with PARITY-SCAN[3] 